\title{
'Why genomes in pieces?' revisited: Sucking lice do their own thing in mtDNA circle game
}

\author{
David M. Rand ${ }^{1}$ \\ Department of Ecology and Evolutionary Biology, Brown University, Providence, Rhode Island 02912, USA
}

About $30 \mathrm{yr}$ ago, an organizational nightmare emerged in the budding field of genomics. Several independent groups discovered that the genetic information encoding genes was not arranged in a continuous linear format of adjacent nucleotides. To everyone's surprise, the coding information for proteins was interrupted by noncoding sequences that were removed from initial RNA transcripts before translation continued the job of the central dogma: information transfer from DNA to RNA to protein. Walter Gilbert made sense of all of this (Gilbert 1978) when, in a few hundred words, he coined the terms intron and exon, predicted that the protein coding content of genomes would comprise only a fraction of the total DNA, triggered the "introns-early" versus "introns-late" debate, and set the seed for the notion that RNA was the early form genetic material.

The field of genomics survived this chaos and now relishes these confusing disruptions, where our understanding of how the world works is turned on its head by some odd fact. In hindsight, there often seems to be an appealing reason for why a genome would engage in something so illogical. Gilbert suggested that introns facilitate higher rates of per-gene recombination, promoting diversity and permitting faster evolutionary change (Gilbert 1978). Some of the biggest questions in genomics today are focusing on phenomena that we might never have imagined only a generation ago: epigenetic modification of expression and transmission, microRNAs and RNA interference, arms of sex chromosomes and autosomes jumping ship and going over to the other side, among others.

One might think that all strange facts of genome organization and expression should have been discovered by now, but even in the heavily scrutinized world of mitochondrial genomics, fascinating novelties are still emerging. The study by Shao et al. (2009) in this issue, reports an almost comical departure from the standard single-circle mitochondrial genome organization found in most animals. It appears that sucking lice associated with humans-and not other lice-have evolved a multiple minichromosome organization for mtDNA that begs the question: Why genomes in pieces? This same question has been asked before in the context of mtDNA organization (Landweber 2007), but animals were not the topic of concern. Departures from the "standard" single-circle mtDNA organization have been observed in protists, such as Diplonema, where genes (and notably parts of genes) are fragmented across many minicircles (Marande and Burger 2007). In the Kinetoplastida (a group including Trypanosomes), mitochondrial genes are distributed among thousands of catenated circles in a network of mini- and maxicircles (for review, see Lukes et al. 2002). Across all of eukaryotic biodiversity, the "standard" single-circle mtDNA may in fact not be the standard, but in animals it is indeed the norm that highlights Shao et al.'s intriguing exception.

'Corresponding author.

E-mail David_Rand@brown.edu; fax (401) 863-2166.

Article is online at http://www.genome.org/cgi/doi/10.1101/gr.091132.109.

\section{The conservative and liberal tendencies of animal mtDNAs}

In the more than half-billion years since the diversification of animals, a tremendous array of body plans has evolved. Nuclear genomes have changed size, base composition, and chromosome composition, among many other evolutionary patterns (Lynch 2007). In contrast, the mitochondrial genomes of animals are one of the most conservative genomic regions in terms of gene content and organization. Animal mtDNA commonly exists as a single, closed, circular molecule ranging from about 15 to $40 \mathrm{~kb}$, with the vast majority in the range of from 15 to $19 \mathrm{~kb}$. The typical bilaterian animal mtDNA contains 37 genes, 13 of which encode subunits of the five complexes of the electron transport chain and ATP synthesis (oxidative phosphorylation, or OXPHOS), 22 of which encode tRNAs, and two of which encode the large and small RNA subunits of the mitochondrial ribosomes (Boore 1999). Moreover, animal mtDNA is commonly very compact: There are generally no introns, little or no intergenic DNA, and most genes show clear signatures of selection for small size.

However, there are accumulating examples of species that deviate from the norm: A coral mtDNA that has a muts gene (Pont-Kingdon et al. 1995); introns have been found in the mtDNAs of sponges (Wang and Lavrov 2008) and sea anemone (Beagley et al. 1998); hydra mtDNA have two linear molecules rather than one circular genome (Pont-Kingdon et al. 2000; Voigt et al. 2008); an onychophoran mtDNA lacks all fourfold degenerate tRNAs (Podsiadlowski et al. 2008); scallop mtDNA shows transposition of certain regions that can more than double genome size (Snyder et al. 1987; Smith and Snyder 2007); and even multicircle organization has been seen in a potato cyst nematode (Armstrong et al. 2000).

Oddly, mtDNA is not conservative at all when it comes to DNA sequence evolution and gene rearrangements within the standard circular molecule. mtDNA typically shows rapid sequence evolution in animals. Many distinct gene arrangements have been observed among the typical set of 37 genes, and tRNAs appear to play a special role in these rearrangements (Gissi et al. 2008). But, the shattering of the sucking lice mtDNA into 18 minichromosomes is a new record for animals. Shao et al. (2009) show that all of the standard 37 mitochondrial genes are present, but each minichromosome carries one to three genes plus a noncoding region with clear sequence conservation across minichromosomes. A strong stem-loop structure is present in the conserved noncoding region of the minichromosomes and may play a role in regulating replication and transcription (a common pattern found in the control-region of single-circle mtDNAs) (Clayton 2003). Using the common structure of the coding + noncoding minichromosome, Shao et al. develop an assay to screen other species of sucking lice that parasitize different parts of the body (head, pubic region, body), as well as those on other primates. When compared with published mtDNAs from other lice species, the minichromosome structure was only found in 
sucking lice species and is curiously common among the species that parasitize humans.

\section{What would Darwin say?}

Why is this mtDNA organization so unusual for animals (and particularly bilaterian animals), while multi-minicircle mtDNA organization is not uncommon among protists? Indeed, there is a bewildering array of mitochondrial genome organizations observed across nonmetazoan eukaryotes (Burger et al. 2003). Despite the greater variation in organization, the patterns of mitochondrial genome structure among microbial eukaryotes does not provide a simple answer to the question: Why genomes in pieces? An intriguing possibility is that parasitic lineages often have reduced and more-derived genome organizations (Burger et al. 2003). The other clear example of a multicircle mtDNA in an animal is in a plant parasite (Armstrong et al. 2000). It is further suggestive that only those lice that feed on blood show the minichromosome organization (Shao et al. 2009), as the other suborders of lice that feed on feathers or hairs have traditional mtDNAs. However, the generality of parasites having unusual mtDNAs is not upheld across a wide range of eukaryotic lineages (Burger et al. 2003). Why a mitochondrial genome may take on a multicircle organization could be explained by two broad kinds of answers: (1) a genome in pieces provides some kind of Darwinian advantage, and (2) genomes in pieces are accidents of molecular genetics and random genetic drift.

One of the hallmarks of animal mtDNA is the lack of recombination (but, see Tsaousis et al. 2005 and references therein), and this is thought to limit its adaptive potential (Rand 2008). With 37 genes spread out across 18 circles, the opportunity for effective recombination is much greater. Novel single nucleotide polymorphisms (SNPs) at one gene can be transmitted more independently of SNPs at other genes, since a population of genespecific circles is transmitted. Linkage disequilibrium between new and existing SNPs should be higher in a single-circle organization, even if multiple mtDNAs exist within a cell. The association of multilocus SNP states across the mtDNA transmitted to an offspring can be different from what was received from one's mother. This enables more independent assortment or effective recombination and, hence, less linkage disequilibrium than the single circle model. Perhaps "genomes in pieces" provided an evolutionary opportunity for sucking lice mtDNA that enabled it to diversify on humans. From an anthropocentric perspective, it is tempting to ascribe something unique to the human parasitism, because we suspect that humans are different from all other organisms. But, Shao et al. (2009) date the emergence of the minichromosome pattern in sucking lice, and it appears that minichromosomes could have predated the evolution of novel human characteristics. Thus, elevated recombination is not likely to have been the driving force behind the transition from one circle to many circles in the putative adaptation of sucking lice to humans.

An alternative explanation for the genomes-in-pieces problem is the greater flexibility of gene expression afforded by a minichromosome organization. Vertebrate mtDNA is transcribed as a single polycistronic message, and the RNAs for individual genes are cleaved from the long RNA molecule by subsequent processing events. This apparently unintelligent design implies that modulation of gene expression may require inefficient whole molecule transcription to vary copy number of individual RNAs. In fact, multiple transcriptional start sites are evident in invertebrate mtDNAs (Cantatore et al. 1990), and recent evidence for multiple sites of RNA-DNA pairing across the mouse mtDNA may enable multiple transcriptional start sites in mouse mtDNA (Brown et al. 2008). But, regulation of expression of multigene transcripts still implies RNA degradation as a mechanism to alter the stoichiometry of transcripts. This costly mechanism could presumably be made more efficient.

With each gene on its own minicircle, modulation of gene expression could be achieved much more effectively by local initiation of transcription. Local control of mitochondrial gene expression is important for modulation of mitochondrial function, and this necessity has been argued as a key reason for why mitochondrial genes remain in the mitochondrion (Wallace 2007). Presumably, it is more efficient to up- or down-regulate local mitochondrial gene expression in targeted regions of the cell than to achieve this by modulation of expression of a class of nuclearencoded genes requiring specific targeting. mtDNA has been retained, so the argument goes, because evolution favors cells, individuals, or lineages that can most efficiently regulate local mitochondrial demands or energy production. But, these molecular arguments in support of Darwinian explanations of genomesin-pieces fall under the weight of their own evidence: If they truly were general explanations for multicircle mtDNA, why is this organization still uncommon in animals? The singular phylogenetic distribution of the sucking lice mtDNA organization could just as well be explained by nonselective arguments (cf. Lynch 2007).

The lice mitochondrial minichromosomes also beg the question of how each circle is faithfully transmitted to the next generation. This seems a relatively simple problem with one kind of mtDNA in the cell. But, if mtDNA bottlenecks in replication and transmission can create variation among daughter cells in the frequency of each mtDNA molecule (e.g., Wai et al. 2008), in the minichromosome circle game, some oocytes could lack critical genes. Presumably, such cells would be eliminated due to compromised OXPHOS, but if copy-number variation could generate fitness variation among oocytes, this system provides an interesting context to study the units of selection on mtDNA (Rand 2001). The current structure of human lice mtDNA implies some history of a race for replication among fragmented minigenomes within the cell, leading to the demise of the traditional mtDNA structure. This apparently Darwinian population biology of competing organelle genomes within the cytoplasm may well have happened, but it is equally possible that this major genome transition was achieved while functional variation at the level of the individual lice was nearly or effectively neutral.

The current study alone cannot answer the question of "Why genomes in pieces?" Despite uncertainty, knowing that a major reorganization of mtDNAs can occur among closely related animals should spur the hunt for answers. It is interesting that significant changes in mitochondrial genome organization or substitution can be observed at relatively fine phylogenetic scales (Gissi et al. 2008). However, major transitions in animal evolution are correlated with changes in mtDNA evolution (Lavrov 2007). What we do not have is an effective means of sorting out the mutation-selection balance for organizational shifts in mitochondrial genomes. If novel mtDNA organizations are observed as short-lived lineages at fine phylogenetic scales, this would support purifying selection against deleterious genome organizations. If clades with novel mtDNA organizations can be observed to be more successful than chance alone, this could support a positive Darwinian role for the mtDNA. The nuclear pieces of this genomic puzzle are certainly part of the solution to this organizational 
nightmare, but there is still a lot we have to learn about mtDNA evolution before we come full circle.

\section{Acknowledgments}

I thank three anonymous reviewers for their comments and acknowledge support from NIH and NSF.

\section{References}

Armstrong, M.R., Blok, V.C., and Phillips, M.S. 2000. A multipartite mitochondrial genome in the potato cyst nematode Globodera pallida. Genetics 154: 181-192.

Beagley, C.T., Okimoto, R., and Wolstenholme, D.R. 1998. The mitochondrial genome of the sea anemone Metridium senile (Cnidaria) Introns, a paucity of tRNA genes, and a near-standard genetic code. Genetics 148: 1091-1108.

Boore, J.L. 1999. Animal mitochondrial genomes. Nucleic Acids Res. 27: $1767-1780$.

Brown, T.A., Tkachuk, A.N., and Clayton, D.A. 2008. Native R-loops persist throughout the Mouse Mitochondrial DNA Genome. J. Biol. Chem. 283: 36743-36751.

Burger, G., Gray, M.W., and Lang, B.F. 2003. Mitochondrial genomes: Anything goes. Trends Genet. 19: 709-716.

Cantatore, P., Roberti, M., Loguercio Polosa, P., Mustich, A., and Gadaleta, M.N. 1990. Mapping and characterization of Paracentrotus lividus mitochondrial transcripts: Multiple and overlapping transcription units. Curr. Genet. 17: 235-245.

Clayton, D.A. 2003. Mitochondrial DNA replication: What we know. IUBMB Life 55: 213-217.

Gilbert, W. 1978. Why genes in pieces? Nature 271: 501. doi: 10.1038/ npg.els.0002804.

Gissi, C., Iannelli, F., and Pesole, G. 2008. Evolution of the mitochondrial genome of Metazoa as exemplified by comparison of congeneric species. Heredity 101: $301-320$.

Landweber, L.F. 2007. Genetics. Why genomes in pieces? Science 318: 405407.

Lavrov, D.V. 2007. Key transitions in animal evolution: A mitochondrial DNA perspective. Integr. Comp. Biol. 47: 734-743.

Lukes, J., Guilbride, D.L., Votypka, J., Zikova, A., Benne, R., and Englund, P.T. 2002. Kinetoplast DNA network: Evolution of an improbable structure. Eukaryot. Cell 1: 495-502.
Lynch, M. 2007. The origins of genome achitecture. Sinauer Associates, Sunderland, MA.

Marande, W. and Burger, G. 2007. Mitochondrial DNA as a genomic jigsaw puzzle. Science 318: 415. doi: 10.1126/science.1148033.

Podsiadlowski, L., Braband, A., and Mayer, G. 2008. The complete mitochondrial genome of the onychophoran Epiperipatus biolleyi reveals a unique transfer RNA set and provides further support for the ecdysozoa hypothesis. Mol. Biol. Evol. 25: 42-51.

Pont-Kingdon, G.A., Okada, N.A., Macfarlane, J.L., Beagley, C.T., Wolstenholme, D.R., Cavalier-Smith, T., and Clark-Walker, G.D. 1995. A coral mitochondrial mutS gene. Nature 375: 109-111.

Pont-Kingdon, G., Vassort, C.G., Warrior, R., Okimoto, R., Beagley, C.T., and Wolstenholme, D.R. 2000. Mitochondrial DNA of Hydra attenuata (Cnidaria): A sequence that includes an end of one linear molecule and the genes for l-rRNA, tRNA(f-Met), tRNA(Trp), COII, and ATPase8. J. Mol. Evol. 51: 404-415.

Rand, D.M. 2001. The units of selection on mitochondrial DNA. Annu. Rev. Ecol. Syst. 32: 415-448.

Rand, D.M. 2008. Mitigating mutational meltdown in Mammalian mitochondria. PLoS Biol. 6: e35. doi: 10.1371/journal.pbio.0060035

Shao, R., Kirkness, E.F., and Barker, S.C. 2009. The single mitochondrial chromosome typical of animals has evolved into 18 minichromosomes in the human body louse, Peduculus humanus. Genome Res. (this issue). doi: 10.1101/gr.083188.108.

Smith, D.R. and Snyder, M. 2007. Complete mitochondrial DNA sequence of the scallop Placopecten magellanicus: Evidence of transposition leading to an uncharacteristically large mitochondrial genome. J. Mol. Evol. 65: 380-391.

Snyder, M., Fraser, A.R., Laroche, J., Gartner-Kepkay, K.E., and Zouros, E. 1987. Atypical mitochondrial DNA from the deep-sea scallop Placopecten magellanicus. Proc. Natl. Acad. Sci. 84: 7595-7599.

Tsaousis, A.D., Martin, D.P., Ladoukakis, E.D., Posada, D., and Zouros, E. 2005. Widespread recombination in published animal mtDNA sequences. Mol. Biol. Evol. 22: 925-933.

Voigt, O., Erpenbeck, D., and Worheide, G. 2008. A fragmented metazoan organellar genome: The two mitochondrial chromosomes of Hydra magnipapillata. BMC Genomics 9: 350. doi: 10.1186/1471-2164-9-350.

Wai, T., Teoli, D., and Shoubridge, E.A. 2008. The mitochondrial DNA genetic bottleneck results from replication of a subpopulation of genomes. Nat. Genet. 40: 1484-1488.

Wallace, D.C. 2007. Why do we still have a maternally inherited mitochondrial DNA? Insights from evolutionary medicine. Annu. Rev. Biochem. 76: 781-821.

Wang, X. and Lavrov, D.V. 2008. Seventeen new complete mtDNA sequences reveal extensive mitochondrial genome evolution within the Demospongiae. PLoS One 3: e2723. doi: 10.1371/journal.pone. 0002723.

\section{Genome Research}




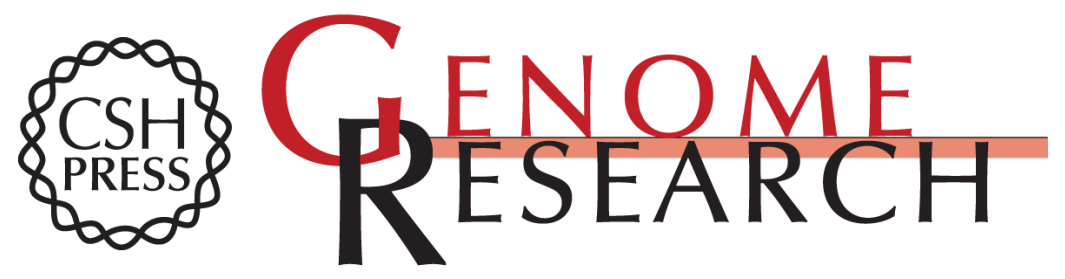

\section{'Why genomes in pieces?' revisited: Sucking lice do their own thing in mtDNA circle game}

David M. Rand

Genome Res. 2009 19: 700-702

Access the most recent version at doi:10.1101/gr.091132.109

Related Content The single mitochondrial chromosome typical of animals has evolved into 18 minichromosomes in the human body louse, Pediculus humanus

Renfu Shao, Ewen F. Kirkness and Stephen C. Barker

Genome Res. May , 2009 19: 904-912

References This article cites 26 articles, 8 of which can be accessed free at: http://genome.cshlp.org/content/19/5/700.full.html\#ref-list-1

Articles cited in:

http://genome.cshlp.org/content/19/5/700.full.html\#related-urls

\section{License}

Email Alerting Receive free email alerts when new articles cite this article - sign up in the box at the Service top right corner of the article or click here.

\section{Affordable, Accurate Sequencing.}

To subscribe to Genome Research go to:

https://genome.cshlp.org/subscriptions 